\title{
Beratung neu gewendet
}

\section{Professionelle Soziale Arbeit erfolgt zumeist in Verhältnissen, mit denen Menschen nicht zurechtkommen}

\author{
Wolf Rainer Wendt
}

If looking for real effects, counselling in Social Work has to challenge the individual who is looking for advice.

Si l'on recherche un réel impact, l'accompagnement en travail social doit relever le défi de pouvoir répondre aux attentes des individus en demande de conseil.

Prof. Dr. Wolf Rainer Wendt ist Vorsitzender der Deutschen Gesellschaft für Soziale Arbeit e. V. Internet http://www.deutschegesellschaft-fuer-soziale-arbeit.de
Beratung in der Sozialen Arbeit muss die Mitwirkung der Ratsuchenden herausfordern, wenn sie nachbaltig wirken soll.

Beratung, die sozialprofessionell geleistet wird, ist eine Reflexions-, Bildungsund Entwicklungsarbeit. Darin besteht ihr sozialer Charakter: Sie hat zum Gegenstand, was in schwieriger Lage zu besorgen ist, und zeichnet sich in der Art und Weise aus, wie wir in solcher Lage mit uns selber und miteinander »zu Rate gehen«. Beratung beinhaltet eine Arbeit an gemeinsamen Belangen oder an Problemen, die nach einer Lösung verlangen. Mit ihnen und mit unser aller Leben wandelt sich auch die Aufgabenstellung für eine soziale Beratung, die sich nicht in einzelnen fachlich erteilten Ratschlägen erschöpft, sondern die individuelle und gemeinsame Lebensführung reflektiert und ihr Perspektiven eröffnet.

Beratung ist das, was Berater zu tun wissen: darüber sind sich Fachkräfte einig. Unter Beratern hält man Beratung für eine Leistung von Experten. Sie hegen und pflegen ihr eigenes Fachgebiet. Sie legen Wert auf die institutionelle Eigenständigkeit und besondere Praxisform dieses Gebiets. Insbesondere therapeutisch orientierte Berater betrachten ihre Beratungsstelle als einen gewissermaßen exterritorialen klinischen Raum, in dem ein von ihnen gestaltetes Regime abgehoben von der Lebenswirklichkeit draußen herrscht.

Professionelle Beratung wird von vielen Diensten im Sozial- und Gesundheitswesen angeboten. Sie wird geliefert von Spezialisten - der Erziehung, der Ernährung, einzelner medizinischer Fachgebiete, der beruflichen Qualifikation, der Psyche, des Drogengebrauchs, sexueller Beziehungen, der Pflege oder der Rehabilitation. Nun wird der Rat von Fachleuten in den genannten Belangen zweifellos gebraucht. In der Sozialen Arbeit greift ihre Expertise aber zu kurz.
In Hinblick auf den professionellen Handlungsauftrag sind Unterscheidungen angebracht: Es gibt Beratungen, die bei Einzelproblemen benötigt werden - von Menschen, denen es ansonsten an Rat im Leben nicht fehlt. Für sie ist Expertise »vorrätig «. Sie wissen diese auch abzurufen, beispielsweise aus dem Internet oder aus anderen Quellen. Und es gibt Beratungen, die für Menschen in prekärer Lage angebracht sind. Dabei handelt es sich um Personengruppen, denen sich die professionelle Soziale Arbeit hauptsächlich widmet. Gesucht ist bei ihnen eine Umsicht in den Verhältnissen, in denen diese Menschen nicht zurechtkommen. Ihre Probleme sind komplex. Einzelne Ratschläge helfen in dieser Lage kaum weiter.

Es gibt neben Einkommensarmut und Bildungsarmut in unserem Informationszeitalter auch so etwas wie eine Armut an Rat. Sie hat mit dem Mangel an Einkommen und Bildung zu tun, ergibt sich aber auch in andauernden Krisen und bei Belastungen, die den Einzelnen überfordern.

In der Sozialen Arbeit sind Menschen mit Rat zu versorgen, die aus vielerlei Gründen mit ihm nicht ausgestattet sind und die ihn sich nicht selber beschaffen können. Sozialer Rat ist heutzutage vom Bürger bei einigem Vermögen (materiell wie immateriell verstanden) auf informellen und formellen Wegen billig zu haben. Bei Unvermögen dagegen ist guter Rat teuer - und will professionell mit Umsicht überlegt sein und vermittelt werden.

Rat wird geschaffen, indem er sich in die persönliche Erfahrung fügt. Verständigen wir uns darüber, wie eine sozial angemessene Beratung zustande kommt, können wir von dem Faktum ausgehen: Menschen beraten sich alltäglich. Sie tauschen Informationen aus und reflektieren ihre Erfahrungen. Sie gehen informell mit sich und Nachbarn, Freunden und Bekannten »zu Rate«; das reicht im Alltag häufig aus. 
Und es gibt fachlich kompetente Stellen, die Beratung anbieten. In bestimmten Situationen sind Menschen "gut beraten ", sich von professioneller Seite beraten zu lassen. Wir haben es mit unterschiedlichen Ausgangspositionen von Beratung zu tun. Ihr Gegenstand sind aber stets Probleme und Situationen, mit und in denen die Ratsuchenden selber zurechtkommen wollen oder müssen.

Zur Erläuterung dieser These können einige begriffliche Klärungen beitragen (vgl. Wendt 2004, 61 ff.). Wer im Leben keinen Rat weiß, kommt nicht weit. Rat begleitet uns überall im Leben, und zur Daseinsbewältigung muss immer wieder Rat hinzukommen. Menschen konsultieren einander. Fachstellen und professionelle Berater ziehen sie "zu Rate « bei speziellen Schwierigkeiten, in denen sie alleine keine Lösung finden. Wenn der Rat, den wir in Fragen der Lebensführung und sozialen Problembewältigung von außen und bei Experten einholen, uns im Alltag weiterhelfen soll, muss er an den lebensbegleitenden Rat anschließen, über den wir schon verfügen. Die dienstliche Beratung sollte um ihrer Nachhaltigkeit willen selber von lebensbegleitender Art sein.

Der Rat der Europäischen Union hat in seiner Entschließung Nr. 9286 am 28. Mai 2004 zum »Ausbau der Politiken, Systeme und Praktiken auf dem Gebiet der lebensbegleitenden Beratung in Europa « hervorgehoben, alle Bürger sollten »in jedem Lebensabschnitt Zugang zu Beratungsdiensten haben, wobei gefährdeten Einzelpersonen und Gruppen in besonderem Maße Rechnung zu tragen ist «. Zwar bezieht sich die Entschließung in erster Linie auf Bildungs- und Berufsberatung, aber mit einem Akzent auf ein lebenslanges Lernen, mit dem die Menschen sich und ihre Kompetenzen an den ständigen Wandel der Anforderungen »im Übergang zu einer wissensbasierten Wirtschaft und Gesellschaft « anpassen können (Europäischer Rat 2004).

Wegleitung und Orientierung kennzeichnen die gemeinte Beratung. Der Verweis auf Wissen und Bildung ist durchaus auch für eine psychosoziale und psychopädagogische Beratung im engeren Sinne angebracht, denn im Beratungsprozess wird die transportierte Information umgewandelt in neues Verständnis. Oder aber ein psychotherapeutisches und psychoedukatives Vorgehen führt Men- schen zu einem Verständnis von sich und ihrer Mitwelt, in welchem Verstehen sie sich neu zu orientieren vermögen.

Das handlungsleitende Prinzip der Kooperation und Koproduktion in der Sozialen Arbeit lässt uns von einem herkömmlichen beratungsberuflichen Verständnis abrücken, wonach Beratung ein Gut ist, das fachlich vorgehalten und von des professionellen Rates bedürftigen Personen (»Beratungsnehmern «) konsumiert wird. Soziale Beratung unterliegt auch als Prozess nicht einfach den Vorgaben und der Expertise von Beratern, sondern wird von den an einer Konsultation Beteiligten gemeinsam unterhalten. Selbst eine breit angelegte, lebenswelt- und alltagsorientierte Definition wie die von Nestmann, Engel und Sickendiek $(2004,599)$ ist in dieser Hinsicht zu korrigieren:

»Beratung ist eine vielgestaltige, sich ständig verändernde und durch viele interne und externe Einflussfaktoren bestimmte professionelle Hilfeform. Sie unterstützt in variantenreichen Formen bei der Bewältigung von Entscheidungsanforderungen, Problemen und Krisen und bei der Gestaltung individueller und sozialer Lebensstile und Lebensgeschichten. «

Die » professionelle Hilfeform « schließt in der Sozialen Arbeit zu Arrangements von Beratung auf, die einzelne Personen, Familien oder Gruppen von sich aus wählen oder auf Anraten und unterstützt von Professionellen gestalten.

Soziale Beratung bleibt auch inhaltlich rückgebunden an Gemeinsamkeiten der sich Beratenden. Bei einem informellen Austausch besteht daran kein Zweifel. Wer sich mit anderen berät, als Frau unter Frauen, Mutter mit Müttern, vertraut einander und unterstellt, dass der andere Beteiligte versteht, um was es geht, und dass er im Gespräch zur Sache etwas beizutragen vermag. Weil Fachleute sich auf vieles, was Betroffene angeht, nicht wirklich verstehen, bietet sich für die Soziale Arbeit ein »Peer Counselling « dergestalt an, dass für Menschen in einer gleichartigen Lebenssituation eine Beratung untereinander organisiert wird: Behinderte konsultieren Behinderte oder Jugendliche leisten, entsprechend geschult, eine Gleichaltrigenberatung in einem Kinderbüro oder einem Jugendtreff.

Beraten Sozialprofessionelle einzelne Personen oder eine Gruppe, suchen sie mit ihnen eine Beratungsbeziehung auf- zubauen, in der sie nicht die Rolle von Experten spielen, sondern von einer auf alle Beteiligten verteilte Expertise ausgehen, mag die Verteilung tatsächlich eine sehr ungleiche sein. Da in der Sozialen Arbeit gewöhnliche Probleme des Daseins und heutiger Lebensführung Gegenstand der Beratung sind, haben an ihnen alle teil - mit mehr oder weniger Vernunft und Empathie, die in der Wahrnehmung der Probleme und der mit ihnen verbundenen Belastungen von den Beteiligten aufgebracht werden.

Fazit: Grundsätzlich leistet Beratung etwas auch ohne fachliche Expertise. Schon zum überkommenen Wortsinn von Beratung (lat. consiliare und consilium) im Deutschen - wie von consulenza im Italienischen, conseiller im Französischen und counselling und consultation im Englischen - gehört der Bedeutungsgehalt, dass sie im Miteinander von Menschen erfolgt, welche zusammen nach einer Problemlösung suchen. Man berät sich gemeinsam: Herkömmlich meint ein Rat eine Mehrzahl von Personen, die sich kundig machen und die kundig sind. Das Wort Beratung steht hier intransitiv für »beratschlagen « (deliberare), wobei man in diesem Vorgang zu einem Beschluss kommt. Gegenstand oder Sache solcher Beratungen ist ein gemeinsamer Rat, der von den Beteiligten eingebracht, eingeholt, ausgetauscht und bearbeitet wird. Im Ergebnis wollen sie "gut beraten « sein.

Zum Prinzip der Kooperation im Beratungsprozess steht nicht im Widerspruch, dass die Kommunikation der Fachkräfte mit den Ratsuchenden asymmetrisch angelegt ist. Der Berater strukturiert und »führt « das Gespräch, präzisiert die Fragestellung, klärt auf und behält die Kontrolle im Verlauf der Kommunikation. Die Ratsuchenden lassen sich darauf ein. Sie versprechen sich von der Asymmetrie in der Struktur institutioneller Beratung, dass sie an der Kompetenz partizipieren, die in der Beratungsstelle vorhanden ist, dass sie in ihr Rat »einholen « können oder ihn - in Form von Information und Wegleitung - auch einfach geliefert bekommen.

Indes ist von professioneller Seite darauf zu sehen, dass sich bei den Adressaten ihrer Bemühungen ein Prozess der Aneignung von Rat vollzieht. Diesen Prozess gilt es zu fördern. Am einfachsten mit der einem Ratschlag hinzugefügten Aufforde- 
rung » Mach was draus! « Menschen in einer prekären Situation - gestresst, verwirrt, gekränkt, gestört - sind aber schwerlich in der Lage, überlegt vorzugehen. Sie müssen in diese Lage erst kommen. Deshalb gehört zur Beratung von derart belasteten Menschen eine weiterführende Soziale Arbeit. Sie erschließt außerhalb der Beratungssituation Ressourcen, sie aktiviert Mitwirkung, vernetzt und unterhält Unterstützung. Eine Beratung ist einzubetten in einen Hilfeprozess, der vorbereitender, komplementärer und nachbereitender Natur ist. In ihm impliziert die Beratungsbeziehung eine professionell $\mathrm{zu}$ unterhaltende Arbeitsbeziehung.

Der Zusammenhang lässt sich von beiden Seiten betrachten. Soziale Arbeit schließt auch dort Beratung ein, wo diese Arbeit nicht in einer Beratungsstelle erfolgt und nicht auf dem Beratungsmarkt extra angeboten wird. Gegen die Tendenz (die in der Deutschen Gesellschaft für Beratung vertreten wird), eine eigenständige Professionalität psychosozialer Beratung zu entwickeln, sei festgehalten, dass in der Sozialen Arbeit überall die Kompetenz zu problemadäquater Beratung verlangt wird.

Die Formulierung »Soziale Arbeit schließt Beratung ein « bedeutet zudem, dass sie ein Kontinuum unterschiedlicher Beratungsformen einbezieht. Ihr Spektrum reicht von der trivialen informellen Beratung, von vielerlei Angeboten der Information und der Orientierung in niedrigschwelligen Anlaufstellen über die fallbezogene professionell organisierte Beratung, fallübergreifende Konsultationen bis zur spezialisierten Beratung etwa im Rahmen Klinischer Sozialarbeit.

In ihrer spezifischen Lebenssituation sind Menschen stets Experten in eigener Sache, und an dieser Expertise mangelt es den Fachkräften durchaus, die in dieser Lage nicht sind. Deshalb liegt es nahe, dass sich von Problemen gleich betroffene Menschen untereinander beraten, wie sie es informell eh schon tun. Formell organisiert geschieht das in vielerlei Selbsthilfegruppen. Indes lässt sich eine Synergie von fachlicher Kompetenz und Selbstkompetenz von »Laien « dadurch erreichen, dass sich mehrere Fachkräfte mit mehreren Ratsuchenden in einer Gruppe themenzentriert zusammenfinden. Das geschieht am besten, wenn die kollegiale Konsultation in einer Beratungsgruppe mit der Gruppenberatung in Selbsthilfe vereinigt wird. Dadurch erweitert sich das Blickfeld von beiden Seiten, die Standpunkte wechseln und in der mehrperspektivischen Sicht lässt sich eine Lösungsstrategie erarbeiten, die dann auch koproduktiv umgesetzt werden kann.

Kompetenzzentren sind passende Orte für eine derartige Konsultation in so zusammengesetzten Beratungsgruppen. Längst gibt es außerhalb der Sozialen Arbeit verschiedene Kompetenzzentren, die eine Bildungs- und Entwicklungsarbeit leisten: berufliche, technologiebezogene, frauenspezifische, interkulturelle Kompetenzzentren, solche für Existenzgründer, für ökologischen Landbau, »für aktive Senioren und bürgerschaftliches Engagement «. Sie alle bieten Gruppenberatung und Einzelberatung an. Sie gemischt vorzuhalten, empfiehlt sich für die Soziale Arbeit, um flexibel dem komplexen Bedarf an Beratung in der Lebensführung von Menschen heutzutage nachzukommen.

Man spricht in diesem Zusammenhang auch von »integrierter Beratung « in dem doppelten Sinne, dass sie einerseits den ganzen Bedarf an sozialer Problembehandlung mit spezifischen Beratungsleistungen abdeckt und dass sie andererseits in einen bedarfsgerechten Prozess der Unterstützung und Bildung einbezogen ist und von ihm nicht getrennt wird. Sie ist niedrigschwellig angelegt und flexibel gestaltet. Vielerorts hat man zum Beispiel die Erziehungsberatungsstellen und andere spezialisierte Beratungsstellen mit dem Allgemeinen Sozialdienst in Beratungszentren verbunden. Den dort zusammengefassten Beratungsangeboten ist eine Orientierungsberatung vorgeschaltet, in der die Professionellen und die Nutzer miteinander klären können, was zu tun ist.

Im Diskurs über die allgemeine Aufgabenstellung von Beratung in der Dynamik des gesellschaftlichen Wandels hat die »Frankfurter Erklärung zur Beratung « im Jahre 2002 die Notwendigkeit einer hohen und dauerhaften Reflexivität festgestellt, und sie erfordere »Orte, an denen diese Reflexivität entwickelt werden kann. Hier ist der Ort für Beratung. «(Forum Beratung 2002, 336)

Soziale Beratung hat eine integrierende Funktion. In ihrem Prozess begegnen und verständigen sich Personen in gemeinsamer Suche nach Lösungen in schwieriger
Lage und nach neuen Wegen. Berater nehmen Anteil, die Beratenen gewinnen Teilhabe, und beider Konsultation sollte so arrangiert werden und organisiert sein, dass die offene Kommunikation anhält, in der die Erfahrung, das Wissen und Wollen und das Können von Menschen in Anbetracht ihrer Probleme ausgetragen wird und sich sozial bearbeiten lässt.

\section{Die Beratungsleistung}

trägt zu eigener Beratung im Leben bei

Rat kann in einer formell gestalteten Kommunikation, auf die ein Beratungsdienst eingerichtet ist, gefunden und gegeben werden. In der Sozialen Arbeit ist diese »stellenweise « Besorgung von Rat möglichst in informelle Beratungsvorgänge einzubetten, in denen sich Menschen stetig informieren und orientieren, sich ihrer Lage und Möglichkeiten vergewissern und sich bilden. Denn eine professionelle Beratung kann nachhaltig nur wirken, wenn sie sich in einer individuellen oder gemeinsamen Selbstverständigung fortsetzt.

Wolf Rainer Wendt

\begin{tabular}{|l} 
Literatur \\
Europäischer Rat: Entschließung über \\
den Ausbau der Politiken, Systeme und \\
Praktiken auf dem Gebiet der lebensbe- \\
gleitenden Beratung in Europa (28. Mai \\
2004). Online: europa.eu.int/comm/ \\
education/policies/2010/doc/coun- \\
cil13832_de.pdf. \\
Forum Beratung der DGVT: Frankfur- \\
ter Erklärung zur Beratung. In: Nest- \\
mann, Frank/Engel, Frank (Hg.): Die \\
Zukunft der Beratung. DGVT, Tübin- \\
gen 2002. S. 335-338. \\
Nestmann, Frank/Engel, Frank/Sicken- \\
diek, Ursel (Hg.): Das Handbuch der \\
Beratung. Band 2: Ansätze, Methoden \\
und Felder. DGVT, Tübingen 2004. \\
Wendt, Wolf Rainer: Rat finden in Ko- \\
operation. Die Soziale Arbeit braucht \\
einen eigenständigen Begriff von Bera- \\
tung. In: Blätter der Wohlfahrtspflege, \\
147, 5+6, 2000. S. 97-99.
\end{tabular}

